\title{
Randomized trial of pragmatic education for low- risk COPD patients: impact on hospitalizations and emergency department visits
}

This article was published in the following Dove Press journal: International Journal of COPD

24 October 2012

Number of times this article has been viewed

\section{Haamid H Siddique' \\ Raymond H Olson ${ }^{2}$ \\ Connie M Parenti ${ }^{2}$ \\ Thomas S Rector ${ }^{2}$ \\ Michael Caldwell ${ }^{3}$ \\ Naresh A Dewan ${ }^{3}$ \\ Kathryn L Rice ${ }^{2}$}

'University of Wisconsin Hospital and Clinics, Madison, WI; ${ }^{2}$ Minneapolis Veterans Affairs Health Care Center, Minneapolis, MN; ${ }^{3}$ Omaha Veterans Affairs Health Care Center, Omaha VA Nebraska-Western, lowa Health Care System, Omaha, NE, USA
Correspondence: Kathryn L Rice Minneapolis Veterans Affairs Health Care Center, I Veterans Drive, Minneapolis MN 55417, USA

Tel + I 6I 246744 I3

Fax + I 6I 27275634

Email kathryn.rice@va.gov
Background: Most interventions aimed at reducing hospitalizations and emergency department (ED) visits in patients with chronic obstructive pulmonary disease (COPD) have employed resource-intense programs in high-risk individuals. Although COPD is a progressive disease, little is known about the effectiveness of proactive interventions aimed at preventing hospitalizations and ED visits in the much larger population of low-risk (no known COPD-related hospitalizations or ED visits in the prior year) patients, some of whom will eventually become high-risk.

Methods: We tested the effect of a simple educational and self-efficacy intervention $(n=2243)$ versus usual care $(n=2182)$ on COPD/breathing-related ED visits and hospitalizations in a randomized study of low-risk patients at three Veterans Affairs (VA) medical centers in the upper Midwest. Administrative data was used to track VA admissions and ED visits. A patient survey was used to determine health-related events outside the VA.

Results: Rates of COPD-related VA hospitalizations in the education and usual care group were not significantly different (3.4 versus 3.6 admissions per 100 person-years, respectively; $95 \% \mathrm{CI}$ of difference -1.3 to $1.0, P=0.77$ ). The much higher patient-reported rates of non-VA hospitalizations for breathing-related problems were lower in the education group (14.0 versus 19.0 per 100 person-years; $95 \%$ CI -8.6 to $-1.4, P=0.006)$. Rates of COPD-related VA ED visits were not significantly different ( 6.8 versus $5.3 ; 95 \% \mathrm{CI}-0.1$ to $3.0, P=0.07$ ), nor were non-VA ED visits (32.4 versus 36.5 ; $95 \% \mathrm{CI}-9.3$ to $1.1, P=0.12$ ). All-cause VA admission and ED rates did not differ. Mortality rates (6.9 versus 8.3 per 100 person-years, respectively; $95 \% \mathrm{CI}-3.0$ to $0.4, P=0.13$ ) did not differ.

Conclusion: An educational intervention that is practical for large numbers of low-risk patients with COPD may reduce the rate of breathing-related hospitalizations. Further research that more closely tracks hospitalizations to non-VA facilities is needed to confirm this finding.

Keywords: chronic obstructive pulmonary disease, education, disease management, selfefficacy

\section{Introduction}

The burden of chronic obstructive pulmonary disease (COPD) on patients and society is substantial. It is the third leading cause of mortality in the USA. ${ }^{1}$ Morbidity from COPD is primarily manifested by daily symptoms with periodic exacerbations, which account for a major portion of the health care costs associated with this disease, particularly when hospitalization is required. ${ }^{2}$

Most patient education and self-efficacy interventions aimed at reducing COPD-related hospitalizations and emergency department (ED) visits are complex, resource-intense programs directed at high-risk patients (ie, patients who have previously 
experienced these events). ${ }^{3-7}$ We recently showed that a comparatively simple, pragmatic COPD case/self-management program in high-risk COPD patients also reduces health care utilization. ${ }^{8}$

Little is known about whether simple educational and selfefficacy interventions reduce COPD-related hospitalizations in low-risk COPD patients (ie, patients who have not had a recent hospitalization or ED visit for COPD). ${ }^{9-13}$ A proactive intervention in low-risk patients may be justified, because a subset of these patients will eventually experience an event that will redefine them as high-risk. Because there are many more low-risk than high-risk COPD patients, and also given that the event rates per individual are lower in this group, practical constraints dictate a broader, less resource-intense approach. We therefore sought to determine if combining simple, pragmatic education with chronic disease management strategies of self-efficacy and goal-setting ${ }^{14,15}$ would reduce the rate of COPD-related ED visits and hospitalizations in a relatively large population of low-risk patients.

\section{Methods}

\section{Study design}

We conducted a randomized controlled trial at three upper Midwest Veterans Affairs Health Care System (VA) centers (VAHCS, Omaha, NE; St Cloud, MN; Minneapolis, MN). Low-risk patients, defined as patients with a spirometrically-confirmed diagnosis of COPD without an ED visit or hospitalization for COPD in the VA in the prior year, ${ }^{13}$ were randomly assigned to limited COPD education or to usual care. The co-primary outcomes were the number of hospitalizations and ED visits for COPD or breathing-related problems over 12 months. The Minneapolis VA Health Care System Institutional Review Board approved the study as Protocol 4140-B for all three sites and waived written informed consent.

\section{Entrance criteria}

Potential subjects were identified through the upper Midwest Veterans Integrated Service Network COPD Chronic Disease Management registry. Inclusion criteria included a clinical diagnosis of COPD, and a predictive forced expiratory volume in 1 second/forced vital capacity (FEV1/FVC) ratio of $<70 \%$ and an $\mathrm{FEV}_{1}$ of $<80 \%$. Exclusion criteria included a COPD exacerbation requiring an ED visit or hospitalization at a VA within the last 12 months, or subject refusal to participate in the study. All patients in the registry who met the study criteria were included in the trial.

\section{Intervention}

Patients were randomly assigned to education or usual care according to an electronically-generated random number table, stratified by site. A locally-developed educational brochure was mailed to patients in the education group. The content of the brochure included topics which were included in the face-to-face education we provided to high-risk COPD patients in our previous study. ${ }^{6}$ The brochure we mailed to low-risk patients included recommendations for smoking cessation, influenza and pneumococcal vaccinations, regular exercise, information about medications for COPD, and information about recognizing and treating COPD exacerbations (see the supplementary materials). In contrast to our previous study, we did not provide face-to-face education or follow-up telephone calls. We also did not provide written COPD exacerbation action plans or prescriptions for action plan steroids or antibiotics; rather, the brochure included a recommendation that patients contact their medical providers to discuss these treatments if they experienced symptoms of COPD exacerbation. In addition, the first mailing included a disease-specific, personal goal-setting questionnaire (smoking cessation, increasing daily activity, eating a healthy diet, losing weight, or receiving an influenza vaccination) (see the supplementary materials), and an option for the patient to decline participation in the study. After 3 months, patients in the education group were mailed a second brochure containing a brief review of the information in the first brochure, as well as local patient testimonials about the benefits of adherence to evidence-based COPD treatment (see the supplementary materials).

\section{Outcomes}

The co-primary outcomes of the trial were the number of hospital admissions and ED visits for COPD within the VA, and the number of self-reported breathing-related hospital admissions and ED visits in non-VA facilities during the 12-month follow up period. Secondary outcomes included hospitalizations and ED visits within the VA due to all causes, all-cause mortality, and patient knowledge about COPD (see the supplementary materials ).

\section{Data collection}

At the end of the 12-month period, data regarding hospitalizations, outpatient visits, and vital status were extracted from national VA data repositories. After 12 months we also mailed a survey to all patients inquiring about any hospitalizations or ED visits for breathing-related problems that had occurred 
in non-VA facilities within the past 12 months. Self-reported data from the latter were analyzed separately from the VA data. A six-question, locally-developed COPD knowledge test, based on the information that was provided in the initial educational brochure, was mailed to both groups at the end of the study period (S2 and S3).

\section{Statistical analysis}

Continuous variables are summarized as means and standard deviations and categorical variables as percentages. Differences in the intervention and usual care group incidence rates during the 12 months of follow-up were analyzed to test the hypotheses that the intervention would reduce hospitalizations and ED visits. Follow-up of participants was censored on known dates of death. Whenever a patient had more than one event, they were assumed to be independent. Having two coprimary endpoints, a $P$-value $\leq 0.025$ was considered statistically significant. Secondary event rates were compared in a similar manner. The number of correct answers on the COPD knowledge questionnaire was compared using Student's $t$-test. Stata software (version 10.1; StataCorp LP, College Station, TX) was used for all analyses. All $P$-values are reported without correction for making multiple comparisons.

\section{Results}

\section{Baseline and follow-up data}

Of the 4425 low-risk patients with spirometrically-confirmed COPD in the registry, 2243 were randomized to the educational intervention and 2182 were randomized to usual care (Figure 1). The baseline characteristics of the two treatment groups were similar (Table 1). Eight patients in the education group requested to be withdrawn from the study, and six were reported deceased by family members in response to our first mailing. Three months later we mailed the second brochure to 2229 patients in the education group. After 12 months, we mailed surveys to 2229 patients in the education group and to 2182 patients in the usual care group.

\section{Primary outcomes}

The primary outcomes of hospitalizations and ED visits due to COPD or breathing-related problems are shown in Table 2. There was a non-significant trend for more COPD-related ED visits in VA facilities in the education group (6.8 visits per 100 person-years compared to 5.3 visits per 100 personyears in the usual care group; $95 \%$ CI of difference -0.1 to 3.0, $P=0.07)$. Rates of COPD-related hospitalizations in VA facilities did not differ (3.4 per 100 person-years in the education group compared to 3.6 visits per 100 person-years in the usual care group; $95 \% \mathrm{CI}$ of difference -1.3 to $1.0, P=0.77$ ). At the end of 1 year, $46 \%$ of the education group and $43 \%$ of the usual care group returned completed surveys regarding breathing-related hospitalizations and ED visits in non-VA facilities. Self-reported hospitalizations for breathing-related problems in non-VA facilities were lower in the education group (14.0 hospitalizations per 100 person-years compared to 19.0 hospitalizations per 100 person-years in the usual care group; $95 \% \mathrm{CI}$ of difference -8.6 to $-1.4, P=0.006$ ). There was a non-statistically significant reduction in self-reported breathing-related ED visits in non-VA facilities in the education group (32.4 visits per 100 person-years compared to 36.5 visits per 100 person-years in the usual care group; 95\% CI of difference -9.3 to $1.1, P=0.12$ ).

\section{All cause ED visits, hospitalizations, and deaths}

As shown in Table 3, there were no differences between the education and usual care groups in all-cause VA hospitalizations or ED visits, respectively (31.3 versus 33.8 hospitalizations per 100 person-years; $95 \%$ CI of difference -6.0 to 1.1 , 44.4 versus $43.5 \mathrm{ED}$ visits per 100 person-years; 95\% CI of difference -3.2 to 5.0). We did not ask patients to report all non-VA admissions or ED visits. There were 141 (6.9) deaths per hundred patient years in the education group and 162 (8.2) deaths per hundred patient years in the usual care group during the 12-month follow-up period $(P=0.13)$.

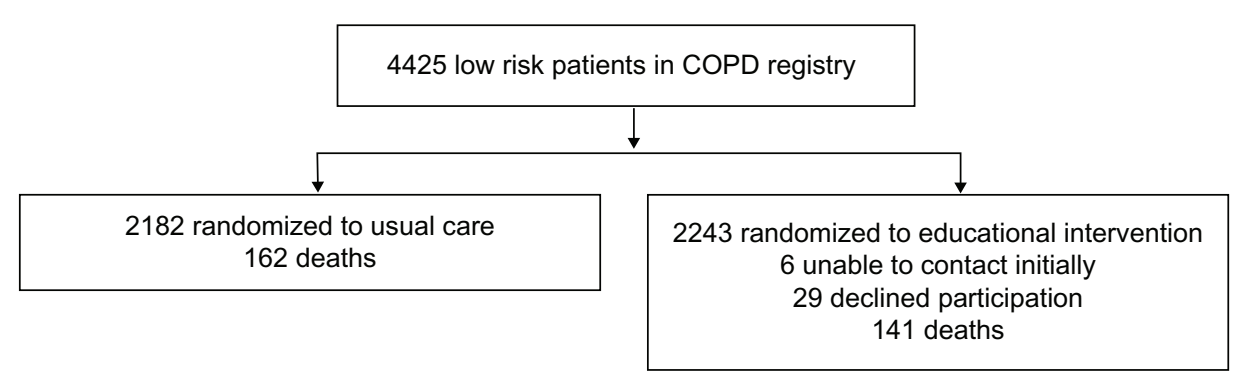

Figure I Study flow diagram. 
Table I Baseline characteristics

\begin{tabular}{lll}
\hline Characteristic & $\begin{array}{l}\text { Education } \\
(\mathbf{n}=\mathbf{2 2 4 3})\end{array}$ & $\begin{array}{l}\text { Usual care } \\
(\mathbf{n}=\mathbf{2 1 8 2})\end{array}$ \\
\hline Mean \pm SD age, years & $70 \pm 10$ & $70 \pm 10$ \\
Male (\%) & $2197(97.9)$ & $2119(97.1)$ \\
Site (\%) & & \\
Minneapolis, MN & $1288(57.4)$ & $1247(57.2)$ \\
Omaha, NE & $571(25.5)$ & $577(26.4)$ \\
St Cloud, MN & $384(17.1)$ & $358(16.4)$ \\
\hline
\end{tabular}

\section{Patient goal setting and COPD knowledge}

A total of $33 \%$ of the patients in the educational intervention group selected a personal COPD-related goal by returning the request mailed to them at the beginning of the trial. At the end of 1 year, 1042 (46\%) in the education group and $931(43 \%)$ of the usual care group completed and returned the COPD knowledge test. COPD knowledge, as indicated by the average number of correct answers to the 6 questions was 4.5 in both groups $(P=0.58)$ with no substantial differences noted for any question.

\section{Discussion}

We tested the effect of a broad-based, pragmatic educational intervention in a relatively large population of lowrisk COPD patients at three VA sites in the USA. Within the VA there were no significant differences in COPDrelated $\mathrm{ED}$ visits or hospitalizations between patients who received COPD education and patients who received usual care. In contrast, COPD education decreased the number of breathing-related hospitalizations that were reported

Table 2 Hospitalizations and ED visits for COPD/breathingrelated problems

\begin{tabular}{|c|c|c|c|c|}
\hline & $\begin{array}{l}\text { Education } \\
(n=2243)\end{array}$ & $\begin{array}{l}\text { Usual care } \\
(n=2182)\end{array}$ & $\begin{array}{l}\text { Difference } \\
(95 \% \mathrm{Cl})\end{array}$ & $P$ value \\
\hline \multicolumn{5}{|l|}{ VA facilities } \\
\hline ED visits & 6.8 & 5.3 & $\begin{array}{l}1.5 \\
(-0.1 \text { to } 3.0)\end{array}$ & 0.07 \\
\hline \multirow[t]{2}{*}{ Hospitalizations } & 3.4 & 3.6 & $\begin{array}{l}-0.2 \\
(-1.3 \text { to } 1.0)\end{array}$ & 0.77 \\
\hline & $n=1042$ & $n=931$ & & \\
\hline \multicolumn{5}{|c|}{ Non-VA facilities } \\
\hline ED visits & 32.4 & 36.5 & $\begin{array}{l}-4.1 \\
(-9.3 \text { to I.I) }\end{array}$ & 0.12 \\
\hline Hospitalizations & 14.0 & 19.0 & $\begin{array}{l}-5.0 \\
(-8.6 \text { to }-1.4)\end{array}$ & 0.006 \\
\hline
\end{tabular}

Note: Results are expressed as rates per 100 person-years ( $95 \% \mathrm{Cl}$ of difference). Abbreviations: ED, emergency department; COPD, chronic obstructive pulmonary disease; VA, Veterans Affairs.
Table 3 Other secondary outcomes

\begin{tabular}{|c|c|c|c|c|}
\hline & $\begin{array}{l}\text { Education } \\
(n=2243)\end{array}$ & $\begin{array}{l}\text { Usual care } \\
(n=2182)\end{array}$ & $\begin{array}{l}\text { Difference } \\
(95 \% \mathrm{Cl})\end{array}$ & $P$ value \\
\hline Mortality & 6.9 & 8.2 & $\begin{array}{l}-1.3 \\
(-3.0 \text { to } 0.4)\end{array}$ & 0.13 \\
\hline $\begin{array}{l}\text { All cause } \\
\text { hospitalizations' }\end{array}$ & 31.3 & 33.8 & $\begin{array}{l}-2.5 \\
(-6.0 \text { to I.I) }\end{array}$ & 0.17 \\
\hline $\begin{array}{l}\text { All cause } \\
\text { ED visits' }\end{array}$ & 44.4 & 43.5 & $\begin{array}{l}0.9 \\
(-3.2 \text { to } 5.0)\end{array}$ & 0.67 \\
\hline $\begin{array}{l}\text { COPD } \\
\text { knowledge }^{2}\end{array}$ & $4.5 \pm 1$ & $4.5 \pm 1$ & 0.0 & 0.58 \\
\hline
\end{tabular}

Notes: 'VA data only. Results are expressed as rates per 100 person-years $(95 \% \mathrm{Cl}$ of difference); ${ }^{2}$ mean \pm standard deviation of number of correct responses.

Abbreviations: ED, emergency department; VA, Veterans Affairs.

in non-VA facilities. There was also a non-statistically significant trend for decreased mortality in the education group.

There is a substantial lack of patient knowledge and understanding of the COPD disease process and treatments. ${ }^{16-20}$ Education has been shown to improve patients' knowledge about COPD, ${ }^{18,19}$ but there is a paucity of information about the effects of simple, limited educational interventions on health care utilization in patients with chronic lung disease. ${ }^{20-25} \mathrm{In}$ a randomized trial of a 4-hour group educational session in 42 patients with mild to moderate COPD, Gallefos reported a reduction in general practitioner visits over a 12-month follow-up period. ${ }^{25}$ In a randomized trial of a mailed educational leaflet in 860 COPD patients, Carré et al showed that the intervention improved patient knowledge, but had no effect on health care utilization after 3 months; hospitalizations and ED visits were not specifically reported. ${ }^{24}$ More information is available in patients with asthma; however, a systematic review of 12 studies of limited education in patients with asthma found only a single study that reported a reduction in ED visits. ${ }^{26}$ Multimodal patient educational interventions are generally thought to be more effective than simple written education, ${ }^{27}$ but we hypothesized that a limited, pragmatic educational intervention in a relatively large number of patients with a longer period of follow-up might be effective in reducing COPD-related ED visits and hospital admissions. We based our educational intervention on our previously successful study in high-risk patients, ${ }^{8}$ but in the current study we emphasized elements we thought would be most pertinent to low-risk patients: lifestyle choices, preventive measures, information about medications, and descriptions of symptoms for which patients should seek further medical attention. Because self-efficacy is considered to be an integral component of self-management of chronic diseases including COPD, ${ }^{14,15}$ we also included patient goal-setting options. 
Most studies examining the effect of COPD patient education on health care utilization have generally incorporated education as a component of resource-intense, comprehensive programs for high-risk patients, usually in conjunction with a chronic disease management or in the setting of a pulmonary rehabilitation program..$^{3-7}$ The educational intervention in this study differed from that of our previous study in high-risk COPD patients, which had included face-to-face education, COPD exacerbation action plans, prescriptions, and follow-up phone calls. Compared to other studies of intensive disease management, our patient population also differed in terms of the severity of their airflow obstruction: the mean $\mathrm{FEV}_{1}$ of low-risk COPD patients in our registry was $1.9 \mathrm{~L}$, compared to $1.0-1.2 \mathrm{~L}$ in the studies conducted by Bourbeau et $\mathrm{al}^{4}$ and Fan et al. ${ }^{7}$

Intensive chronic disease management was associated with unexpected excess mortality in the recent study by Fan et al..$^{7}$ The authors could not discern the reason for the excess mortality, although they noted that their intervention failed to have an impact on patient behavior with regard to respiratory medication usage, including prednisone and antibiotics for $\mathrm{COPD}$ exacerbations. In a recent meta-analysis by Hurley et $\mathrm{a}^{28}$ of ten COPD disease management trials, the excess mortality in the study by Fan et al was an outlier. ${ }^{7}$ Nonetheless, we believe that it is important to carefully monitor the effects of educational interventions in COPD, including mortality and other unanticipated adverse events.

Disease management programs in high-risk COPD patients have, of necessity, included relatively small numbers of patients because of their resource-intense nature; yet high-risk patients represent only a small fraction of the total population of patients. Over $90 \%$ of the COPD patients in the upper Midwest VAHCS Chronic Disease COPD registry are classified as low-risk, (ie, lacking a COPD-related ED visit or hospitalization within the VAHCS in the past 12 months). We did not perform a formal cost-benefit analysis, but our results suggest that a proactive, practical, low-cost educational intervention in a large number of low-risk patients might be costeffective by reducing respiratory-related hospitalizations.

Patients' basic knowledge of COPD at the end of the 12-month study period was not better in the education group, and thus does not explain the observed reduction in patientreported hospitalizations in non-VA facilities.

\section{Limitations}

Our study has certain limitations. Consistent with the current demographics of patients receiving care in the VA, our patients were almost exclusively male. We did not ascertain how many patients actually received or read the brochure. The registry we used to identify low-risk COPD patients does not include hospitalizations and ED visits that occurred outside of the VA, so some high-risk patients may have been included in the sample, although the randomization of a large number of patients is expected to balance the risk profiles, and the potential existence of such unidentified patients may provide further justification for this intervention. Fewer than half of the patients in each group responded to the final survey, and we have no information about events that may have occurred outside the VA rates in non-responders. The incidence of breathing-related ED visits and hospitalizations outside the VA was considerably higher than that within the VA, but the relatively modest response rate to the survey limits the strength of this observation, which may be biased. Patients who received the education may have been more discriminating when attributing hospital admissions to breathing problems; however, the recall of major medical events among the respondents may have been better than among the usual care group. Finally, although our intervention had no apparent effect on patients' knowledge of COPD, the questionnaire was brief and was not validated, and this may not have accurately reflected patients' understanding of their disease.

\section{Conclusion}

A practical educational intervention incorporating principles of chronic disease management may reduce the rate of breathing-related hospitalizations in the large proportion of patients with COPD who are at relatively low-risk for such events. This effect appeared to be due to a reduction in hospitalizations outside of the VA, where most of the hospitalizations occurred. Further research that more closely tracks hospitalizations in non-VA facilities is needed to confirm this finding. This proactive approach may be justified, especially if it can be shown to be cost-effective.

\section{Funding}

Clinical trials.gov NCT00932711. This research was funded by a grant from Veterans Affairs Veterans Integrated Service Network 23, with support from the Minneapolis Veterans Affairs Health Care Center Research Service, and the Minneapolis Veterans Affairs Health Care Center for Chronic Disease Outcomes Research.

\section{Acknowledgments}

Dr Siddique contributed to the conception and design of the study, interpreted the data, and drafted and revised the article critically for important intellectual content. Mr Olson 
contributed to data acquisition, conception and design of the study, interpreted the data, and revised the article critically for important intellectual content. Dr Parenti contributed to the conception and design of the study, interpreted the data, and revised the article critically for important intellectual content. Dr Rector contributed to the conception and design of the study, analyzed and interpreted the data, and revised the article critically for important intellectual content. Mr Caldwell contributed to the conception and design of the study, interpreted the data, and revised the article critically for important intellectual content. Dr Dewan contributed to the conception and design of the study, interpreted the data, and revised the article critically for important intellectual content. Dr Rice had full access to all the data in the study and takes responsibility for the integrity of the data and the accuracy of the data analysis, contributed to the conception and design of the study, interpreted the data, and drafted and revised the article critically for important intellectual content. We are grateful to research assistant Neil Henriksen for the coordination of brochure mailings and data collection; Mary Dahlheimer RN and Catherine Kane, RN, for advice about COPD case/self management; Melissa Partin, $\mathrm{PhD}$, for advice about the educational brochures; and Janet Murphy, MBA, Director, VISN 23, for ongoing support of the VISN 23 COPD Chronic Disease Management Initiative. The sponsors had no role in the design of the study, the collection and analysis of the data, or in the preparation of the manuscript.

\section{Disclosure}

The authors report no conflicts of interest in this work. This work was performed at the Minneapolis Veterans Affairs Health Care Center, Minneapolis MN; the St Cloud Veterans Affairs Health Care Center, St Cloud MN; and the Omaha Veterans Affairs Health Care Center, VA Nebraska-Western Iowa Health Care System.

\section{References}

1. Leading causes of death [webpage on the Internet]. Hyattsville, MD: Centers for Disease Control and Prevention; 2012 [updated January 27, 2012]. Available from: http://www.cdc.gov/nchs/fastats/lcod.htm/. Accessed August 8, 2012.

2. Strassels SA, Smith DH, Sullivan SD, Mahajan PS. The costs of treating COPD in the United States. Chest. 2001;119(2):344-352.

3. Adams SG, Smith PK, Allan PF, Anzueto A, Pugh JA, Cornell JE. Systematic review of the chronic care model in chronic obstructive pulmonary disease prevention and management. Arch Intern Med. 2007; 167(6):551-561.

4. Bourbeau J, Julien M, Maltais F, et al; for Chronic Obstructive Pulmonary Disease axis of the Respiratory Network Fonds de la Recherche en Santé du Québec. Reduction of hospital utilization in patients with chronic obstructive pulmonary disease: a disease-specific self-management intervention. Arch Intern Med. 2003;163(5):585-591.
5. Effing T, Monninkhof EM, van der Valk PD, et al. Self-management education for patients with chronic obstructive pulmonary disease. Cochrane Database Syst Rev. 2007;4:CD002990.

6. Peytremann-Bridevaux I, Staeger P, Bridevaux PO, Ghali WA, Burnand B. Effectiveness of chronic obstructive pulmonary disease-management programs: systematic review and meta-analysis. Am J Med. 2008;121(5):433-443. e4.

7. Fan VS, Gaziano JM, Lew R, et al. A comprehensive care management program to prevent chronic obstructive pulmonary disease hospitalizations: a randomized, controlled trial. Ann Intern Med. 2012; 156(10):673-683.

8. Rice KL, Dewan N, Bloomfield HE, et al. Disease management program for chronic obstructive pulmonary disease: a randomized controlled trial. Am J Respir Crit Care Med. 2010;182(7):890-896.

9. Blackstock F, Webster K. Disease-specific health education for COPD: a systematic review of changes in health outcomes. Health Educ Res. 2007;22(5):703-717.

10. Worth H, Dhein Y. Does patient education modify behaviour in the management of COPD? Patient Educ Couns. 2004;52(3):267-270.

11. Decramer M, Miravitlles M, Price D, et al. New horizons in early stage COPD - improving knowledge, detection and treatment. Respir Med. 2011;105(11):1576-1587.

12. Harris M, Smith BJ, Veale A. Patient education programs - can they improve outcomes in COPD? Int $J$ Chron Obstruct Pulmon Dis. 2008;3(1):109-112.

13. Niewoehner DE, Lokhnygina Y, Rice K, et al. Risk indices for exacerbations and hospitalizations due to COPD. Chest. 2007;131(1): $20-28$.

14. Wagner EH. Chronic disease management: what will it take to improve care for chronic illness? Eff Clin Pract. 1998;1(1):2-4.

15. Davis AH, Carrieri-Kohlman V, Janson SL, Gold WM, Stulbarg MS. Effects of treatment on two types of self-efficacy in people with chronic obstructive pulmonary disease. J Pain Symptom Manage. 2006;32(1):60-70.

16. Scott AS, Baltzan MA, Dajczman E, Wolkove N. Patient knowledge in chronic obstructive pulmonary disease: back to basics. COPD. 2011;8(5):375-379.

17. Hernandez P, Balter M, Bourbeau J, Hodder R. Living with chronic obstructive pulmonary disease: a survey of patients' knowledge and attitudes. Respir Med. 2009;103(7):1004-1012.

18. Barr RG, Celli BR, Mannino DM, et al. Comorbidities, patient knowledge, and disease management in a national sample of patients with COPD. Am J Med. 2009;122(4):348-355.

19. Hyland ME, Jones RC, Hanney KE. The Lung Information Needs Questionnaire: Development, preliminary validation, and findings. Respir Med. 2006;100(10):1807-1816.

20. White R, Walker P, Roberts S, Kalisky S, White P. Bristol COPD Knowledge Questionnaire (BCKQ): testing what we teach patients about COPD. Chron Respir Dis. 2006;3(3):123-131.

21. Scala D, Cozzolino S, D'Amato G, et al. Sharing knowledge is the key to success in a patient-physician relationship: how to produce a patient information leaflet on COPD. Monaldi Arch Chest Dis. 2008;69(2): 50-54.

22. Hill K, Mangovski-Alzamora S, Blouin M, et al. Disease-specific education in the primary care setting increases the knowledge of people with chronic obstructive pulmonary disease: a randomized controlled trial. Patient Educ Couns. 2010;81(1):14-18.

23. Brough FK, Schmidt CD, Rasmussen T, Boyer M. Comparison of two teaching methods for self-care training for patients with chronic obstructive pulmonary disease. Patient Couns Health Educ. 1982;4(2):111-116.

24. Carré PC, Roche N, Neukirch F, et al. The effect of an information leaflet upon knowledge and awareness of COPD in potential sufferers. A randomized controlled study. Respiration. 2008;76(1):53-60.

25. Gallefoss F. The effects of patient education in COPD in a 1-year follow-up randomised, controlled trial. Patient Educ Couns. 2004;52(3): 259-266.

26. Gibson PG, Powell H, Coughlan J, et al. Limited (information only) patient education programs for adults with asthma. Cochrane Database Syst Rev. 2002;2:CD001005. 
27. Trevena LJ, Davey HM, Barratt A, Butow P, Caldwell P. A systemic review on communicating with patients about evidence. J Eval Clin Pract. 2006;12(1):13-23.
28. Hurley J, Gerkin RD, Fahy B, Robbins RA. Meta-analysis of self-management education for patients with chronic obstructive pulmonary disease. Southwest Journal of Pulmonary and Critical Care. 2012;4: 194-202. 


\section{Supplementary materials}

\section{SI Initial educational brochure}

COPD is a lung disease that makes breathing difficult. Airways get tight, swell up and can make more sputum/ phlegm.

COPD stands for "Chronic Obstructive Pulmonary Disease"

Chronic means won't go away

Obstructive means partly blocked

Pulmonary means lungs

Disease means sickness

People who smoke should quit.

Quitting smoking helps the medicine for COPD work better and helps breathing get better.

Talk to your doctor if you want help to quit smoking. You may also call 1-800-QUIT NOW (784-8669).

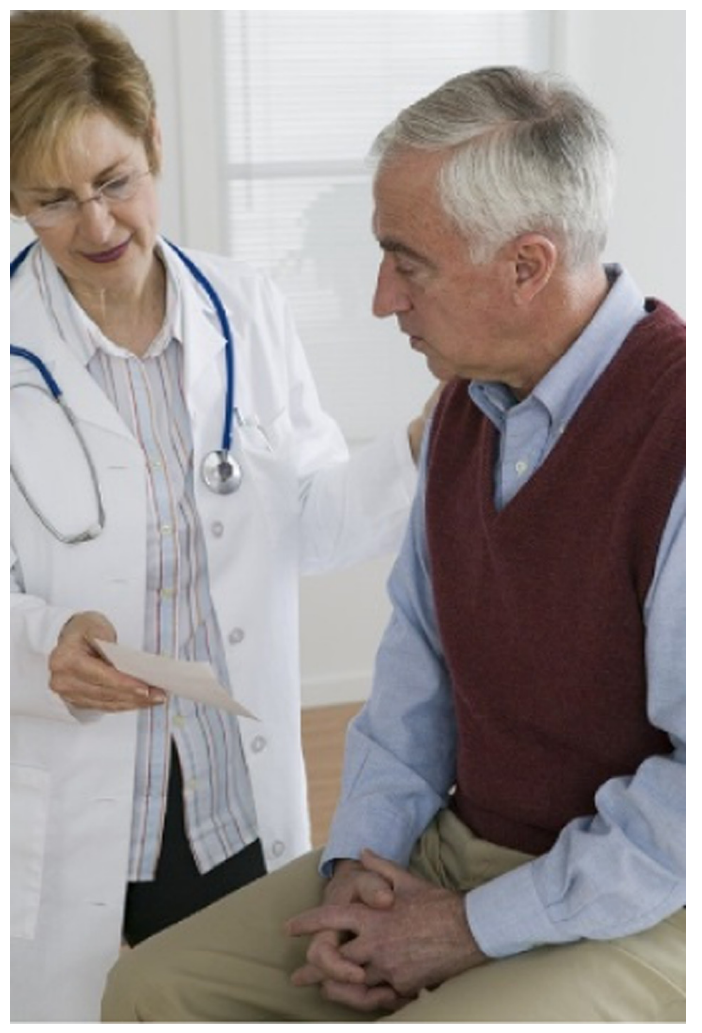

To help stay healthy, make sure to do these things:

- Take your breathing medicines the way your provider has ordered

- Get your flu shot every year

- Get a pneumococcal vaccination at least once

- Wash your hands or use hand sanitizer when out in public

- Eat a balanced diet
- Try to walk or be active at least a total of 20-30 minutes a day

Inhalers are medications that help make your breathing better. Some inhalers your provider may give you are:

- Albuterol is a short acting rescue inhaler that you should always keep with you. The usual dose is 2 puffs 4 times a day as needed for breathing.

- Combivent is two short acting medicines in one, albuterol + ipratropium bromide. The usual dose is 2 puffs 4 times a day.

- Formoterol (Foradil) is a long acting inhaler and is inhaled twice a day.

- Tiotropium (Spiriva) is another long acting inhaler and is inhaled once a day.

The following inhalers have a small amount of steroid medicine in them. They help in reducing swelling in your airways and help your breathing in the long run. It is important to rinse your mouth with water after each use of these inhalers.

- Mometasone (Asmanex) is a steroid inhaler that helps reduce swelling in your airways. The usual dose is 2 times a day.

- Fluticasone (Flovent) is another long acting steroid inhaler that is taken twice a day.

- Advair is a combination inhaler. It is a steroid and a bronchodilator that is taken twice daily.

- Flunisolide (Aerobid) is another steroid inhaler that is used twice daily. Again, like all steroid inhalers, rinse your mouth after each use.

If your sputum/phlegm changes from what it normally looks like, to yellow, green, or gets thicker than normal, you may have an infection. Contact your provider, who may prescribe an antibiotic to treat the infection. If so, take it the way it is ordered. Let your provider know if you think it isn't helping.

Sometimes COPD patients get much more short of breath than usual. If that happens to you, contact your provider. He or she may decide to prescribe a short course (1-2 weeks) of prednisone, a steroid medicine to help your breathing. Let your provider know if you think it isn't helping.

\section{Remember, for a Medical Emergency Dial 911}

Or

Call the toll free VA NURSE Telephone advice line at 1-866687-7382. Available evenings, nights, and 24 hours/day on weekends and Federal holidays. 
Prescription refill number toll free 1-800-661-0827

Content approval: Pulmonary Section

August 2006

Revised February 2007

Revised March 2007

Revised January 2007

Revised July 2009

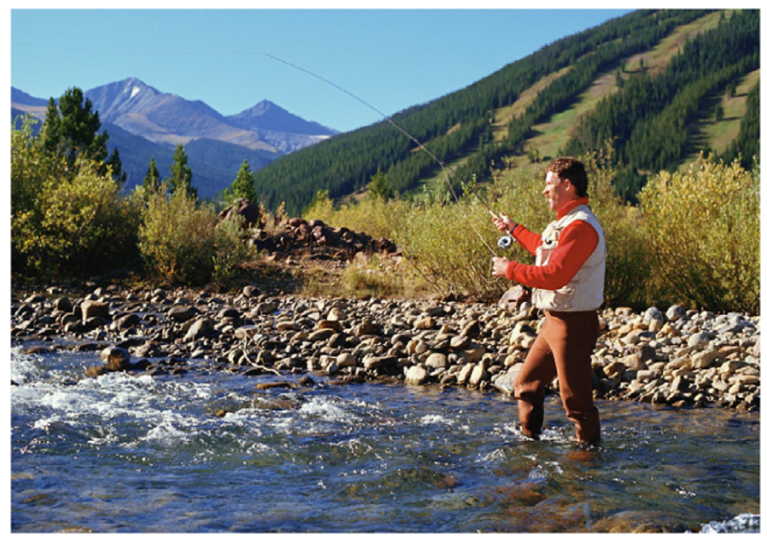

\section{Living Better with COPD}

Minneapolis VA Medical Center

Minneapolis, Minnesota

\section{S2 COPD goal setting questionnaire}

We encourage you to set a goal for yourself to improve your health.

Please place a check mark in front of one or more of the choices below:

Quit smoking

Increase my daily activity

Eat a healthy diet

Lose weight

Get a flu shot this year

Other (please fill in below if you have another goal)

None

Please return this questionnaire in the enclosed envelope

\section{S3 3 month Educational brochure}

COPD is a lung disease that makes it hard to breathe. Airways get tight, swell up and can make more sputum/ phlegm.

VA patients being treated for COPD have said:
"I take my breathing medications every day like they're prescribed, and I feel good, and breathe good."

"My breathing is much better since I have been using my inhalers every day."

"I'm not having any problems since I stopped smoking, and started taking all my meds."

"I'm not short of breath as often because I'm sticking with my medication routine, and trying to quit smoking."

"Walking every day has made me feel better."

"I'm a lot better, my breathing is pretty good. I'm walking a heckuva lot more, and eating more vegetables."

"I've improved since I came to the VA. I've been using exercises in the booklet. I relax and practice deep breathing."

"I wash my hands and carry hand sanitizer with me. I don't want to get sick again.”

To help stay healthy, make sure to do these things:

- Take your breathing medicines as prescribed

- Wash your hands or use hand sanitizer when out in public

- Try to walk or be active for at least 20-30 minutes a day

- Eat a healthy, nutritious diet

- Get a flu shot every year

- Get a pneumococcal vaccination at least once

- Contact your doctor if your sputum/phlegm changes. You may need an antibiotic

- Contact your doctor if you are more short of breath than usual. You may need a different medication

- Quit smoking. If you need help quitting, talk to your doctor or call 1-800-QUIT NOW (784-8669).

If you get much more short of breath than usual, call your health care provider. S/he may decide to prescribe a short course ( 1 to 2 weeks) of prednisone. Prednisone is a steroid medicine that will help your breathing. Tell your health care provider if it doesn't help.

\section{If you have a Medical Emergency, call 911}

If you are not getting better or have any questions please call the Call Center at: (612) 467-1100 or 1-866-414-5058, extension 1100 (toll-free), Monday-Friday, between 8:00 am and 4:30 pm.

At any other time, call the Nurse VA Line at 1-866-6877382 (toll-free).

The prescription refill number is 1-800-661-0827

Content approval: Pulmonary Section

June 2009

VAPE $06 / 2009$ 


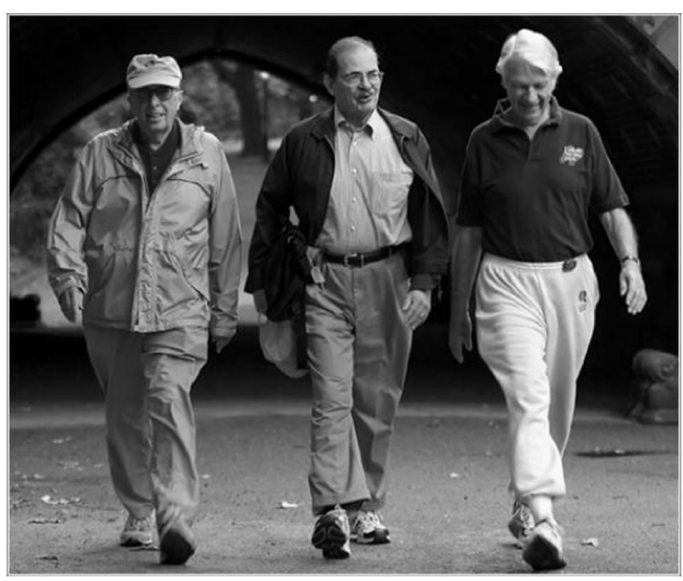

Living Better With COPD

Here's what other veterans have to say ...

"Using my inhalers really has helped"-

Minneapolis VA veteran

Minneapolis VA Medical Center

Minneapolis, Minnesota

Name:

(Last Name)

(First Initial)

Last Four of Social Security Number:

\section{S4 COPD knowledge test COPD questionnaire}

Please circle the number which is next to the answer which you think is correct

\section{Q.1. COPD is}

1. an abbreviation for a VA agency

2. a chronic lung disease
3. a form of arthritis

Q.2. The most important step you can take to help treat your COPD is

1. eat a high carbohydrate diet

2. decrease alcohol intake

3. quit smoking

Q.3. is a long acting inhaler used to treat COPD that should be taken twice a day.

1. albuterol

2. formoterol

3. prednisone

Q.4. Which of these are useful for folks with COPD to stay healthy?

1. Getting a flu shot every year

2. Trying to be as active as possible

3. Taking your breathing medicine the way it has been prescribed

4. All of these

Q.5. If your sputum changes to a yellow or green color or becomes thicker than normal,

1. it is a side effect of your inhalers and you should stop using them

2. you are getting dehydrated and should drink lots of liquids

3. you may have an infection and should contact your provider who may prescribe an antibiotic

Q.6. Prednisone is a medicine that is sometimes used

1. to treat shortness of breath in patients with COPD

2. to treat diabetes in patients with COPD

3. to treat infections in COPD

\section{Publish your work in this journal}

The International Journal of COPD is an international, peer-reviewed journal of therapeutics and pharmacology focusing on concise rapid reporting of clinical studies and reviews in COPD. Special focus is given to the pathophysiological processes underlying the disease, intervention programs, patient focused education, and self management protocols.

\section{Dovepress}

This journal is indexed on PubMed Central, MedLine and CAS. The manuscript management system is completely online and includes a very quick and fair peer-review system, which is all easy to use. Visit http://www.dovepress.com/testimonials.php to read real quotes from published authors. 